\title{
Polynomial Weyl Representations
}

\author{
By
}

\author{
Paul L. ROBINSON*
}

\begin{abstract}
For the canonical commutation relations in infinite dimensions, we offer an explicit direct construction of Weyl representations generated from the Fock representation by polynomial transformations of arbitrary degree, solving a problem posed by Proksch, Reents and Summers. Our solution employs new approaches to Hilbert-Schmidt polynomials and their Wick ordering.
\end{abstract}

\section{Introduction}

Representations of the canonical commutation relations (or CCR) provide a standard mathematical framework in which to discuss bosonic systems. There are two types of such representations associated to a real vector space $V$ equipped with a symplectic form $\Omega$. A Weyl representation of the CCR over $(V$, $\Omega$ ) associates to each $v \in V$ a unitary (Weyl) operator $W(v)$ on some complex Hilbert space $\mathbb{H}$ satisfying the Weyl relations

$$
v_{1}, v_{2} \in V \Rightarrow W\left(v_{1}\right) W\left(v_{2}\right)=\exp \left[\frac{\Omega\left(v_{1}, v_{2}\right)}{2 i \hbar}\right] W\left(v_{1}+v_{2}\right)
$$

and having the property that each one-parameter unitary group $(W(t v): t \in \mathbb{R})$ is strongly continuous. A Heisenberg representation of the CCR over $(V, \Omega)$ associates to each $v \in V$ a self-adjoint (field) operator $\Phi(v)$ in some complex Hilbert space $\mathbb{H}$ satisfying the Heisenberg relations

$$
v_{1}, v_{2} \in V \Rightarrow\left[\Phi\left(v_{1}\right), \Phi\left(v_{2}\right)\right] \subset i \Omega\left(v_{1}, v_{2}\right) I
$$

together with conditions that cope with the unboundedness of the field operators. Passage from Weyl form to Heisenberg from assigns to $(W(t \sqrt{\hbar} v): t$ $\in \mathbb{R})$ its self-adjoint infinitesimal generator $\Phi(v)$ so that $W(\sqrt{\hbar} v)=$ $\exp [i \Phi(v)]$; passage from Heisenberg form to Weyl form is via exponentiation when possible. See [2].

Communicated by T. Kawai, November 20, 1997.

1991 Mathematics Subject Classifications: 81SO5, 46B28, 33C50, 28C20

*Department of Mathematics, University of Florida, Gainesville FL32611, U.S.A. 
The case most commonly considered is that in which $V$ is the real vector space underlying a complex Hilbert space and $\Omega$ is the imaginary part of the complex inner product. In this case, there are several thoroughly studied classes of representations of the CCR over $(V, \Omega)$. Most familiar of these is the Fock representation, which comes in a number of equivalent versions and is suitable for describing a free boson field. Less familiar but also well studied are the coherent representations and the quasifree representations; both classes arise when Fock representations are subjected to certain standard operations. Recently, a new class of representations of the CCR over $(V, \Omega)$ was introduced and studied by Proksch, Reents and Summers [7]. These are called quadratic representations of the CCR: as originally constructed, they arise when Fock field operators are subjected to quadratic transformations; the resulting Heisenberg representations actually exponentiate to yield Weyl representations. In [8] we presented a direct construction of these quadratic Weyl representations, entirely circumventing the Heisenberg representations and so obviating the need to deal with unbounded operators.

Our primary aim in this paper is to present a uniform direct construction of Weyl representations generated from the Fock representation by means of polynomials of arbitrary degree; this solves a problem raised by Proksch, Reents and Summers. We have two secondary aims related to the technical machinery involved in our construction: the one is to offer a new approach to Hilbert-Schmidt polynomials; the other is to offer a new approach to the Wick-ordering of such polynomials. Our primary aim is likely to be of greatest interest to quantum field theory; in particular, the nonlinearity inherent to polynomial Weyl representations suggests that they might play a rôle in the construction of interacting fields. Our secondary aims are likely to be of interest more generally, for example in functional analysis and stochastic analysis.

The following brief outline of our construction may be helpful as orientation. Considering first the finite-dimensional situation, let $\mathbb{R}^{m}$ have the standard inner product and let $\mathbb{R}^{2 m}$ have the symplectic form given by

$$
\Omega\left(x_{1} \oplus y_{1}, x_{2} \oplus y_{2}\right)=\left(x_{1} \mid y_{2}\right)-\left(y_{1} \mid x_{2}\right)
$$

where $x_{1}, x_{2}, y_{1}, y_{2} \in \mathbb{R}^{m}$. The Schrödinger representation is the Weyl representation $W_{0}$ of $\mathbb{R}^{2 m}$ on the space $\mathbb{L}^{2}\left(\mathbb{R}^{m}\right)$ of all complex functions on $\mathbb{R}^{m}$ squareintegrable relative to Lebesgue measure, determined by the rule that if $f$ $\in \mathbb{L}^{2}\left(\mathbb{R}^{m}\right)$ and $x, y, z \in \mathbb{R}^{m}$ then

$$
\left[W_{0}(x \oplus y) f\right](z)=\exp \left[\frac{(x \mid 2 z-y)}{2 i \hbar}\right] f(z-y) .
$$

Let $\Lambda: \mathbb{R}^{m} \rightarrow \mathbb{R}$ be a polynomial (or more generally measurable) function: define a unitary operator $U_{\Lambda}$ on $\mathbb{L}^{2}\left(\mathbb{R}^{m}\right)$ by 


$$
\left[U_{A} f(\boldsymbol{z})=\exp \left[\frac{\Lambda(z)}{i \hbar}\right] f(\boldsymbol{z})\right.
$$

for $f \in \mathbb{L}^{2}\left(\mathbb{R}^{m}\right)$ and $z \in \mathbb{R}^{m}$; write $\Lambda^{u}(z)=\Lambda(u+z)-\Lambda(z)$ for $u$ and $z$ in $\mathbb{R}^{m}$. The rule

$$
x, y \in \mathbb{R}^{m} \Rightarrow W_{\Lambda}(x \oplus y)=U_{\Lambda} W_{0}(x \oplus y) U_{\Lambda}^{-1}
$$

plainly defines a Weyl representation, equivalently given by the rule

$$
x, y \in \mathbb{R}^{m} \Rightarrow W_{\Lambda}(x \oplus y)=W_{0}(x \oplus y) U_{\Lambda^{y}} .
$$

For passage to infinite dimensions, the Schrödinger representation is replaced by the version of the Fock representation called the renormalized Schrödinger representation on $Q$-space, in which the Lebesgue measure is replaced by a consistent family of Gaussian probability measures on finite-dimensional subspaces. In order for the polynomial function $\Lambda$ to define (as a strong limit) a unitary operator $U_{\Lambda}$ on $Q$-space, it should be of Hilbert-Schmidt class (as a growth condition) and Wick-ordered (relative to the Gaussian measures). Notice that if $\Lambda$ is a degree $d+1$ homogeneous polynomial then $\Lambda^{u}$ is a polynomial of degree at most $d$ for each vector $u$; consequently, requiring that $\Lambda$ be Hilbert-Schmidt is more stringent than requiring that each $\Lambda^{u}$ be Hilbert-Schmidt. Accordingly, we assume of the polynomial $\Lambda$ that each $\Lambda^{u}$ is Hilbert-Schmidt and define a polynomial Weyl representation $W_{\Lambda}$ by the infinite-dimensional analogue of $(*)$.

In Section I we offer an essentially self-contained account of HilbertSchmidt polynomials. Our account differs from conventional ones: for example, Hilbert-Schmidt polynomials and their norms are deliberately defined without reference to orthonormal bases, thereby avoiding the need to check independence of choices. The approach may be tailored to suit Hilbert-Schmidt multilinear forms, Hilbert-Schmidt operators and so on. A more conventional account (incorporating boundedness as a hypothesis) may be found in [6]. In Section II we offer a largely self-contained account of Wick-ordering for Hilbert-Schmidt polynomials. Our account here is also out of the ordinary: for example, both the Wick transform and its inverse are defined without reference to orthonormal bases. For accounts of $Q$-space and the renormalized Schrödinger representation, we refer to [2] [5] [9] [10] [12]. In Section III we construct the polynomial Weyl representations of our title, illustrating the construction by examples in which the polynomial has low degree. If the polynomial $\Lambda$ is $(d+1)$-homogeneous, then when $d<2$ the representation $W_{\Lambda}$ arises from the renormalized Schrödinger representation by an inhomogeneous linear canonical transformation while when $d=2$ the representation $W_{\Lambda}$ is quadratic as in [7] and [8]; when $d>2$ the representation $W_{\Lambda}$ is new. Finally, we discuss briefly certain issues stemming from our construction. 
The author is grateful to Martin Florig and Steve Summers for informative conversations and would like to point out that Martin Florig has an independent construction of polynomial representations of the canonical commutation relations based on the infinitesimal approach developed by Proksch, Reents and Summers.

\section{Hilbert-Schmidt Polynomials}

Let $L$ be a real Hilbert space having $(\cdot \mid \cdot)$ as its inner product. By a $d$-homogeneous (real) polynomial $\Lambda: L \rightarrow \mathbb{R}$ we mean the composite of a symmetric multilinear $d$-form $\underline{\Lambda}: L \times \cdots \times L \rightarrow \mathbb{R}$ with the diagonal map from $L$ into the product of $d$ copies, so

$$
z \in L \Rightarrow \Lambda(z)=\underline{\Lambda}(z, \cdots, z) .
$$

The principle of polarization serves to recover $\Lambda$ from $\Lambda$ : explicitly, if $z_{1},{ }^{\cdots,}, z_{d} \in$ $L$ and summation extends over all $2^{d}$ respective sign choices then

$$
2^{d} d ! \Lambda\left(z_{1}, \cdots, z_{d}\right)=\sum_{ \pm \cdots \pm} \pm \cdots \pm \Lambda\left( \pm z_{1} \cdots \pm z_{d}\right) .
$$

Let $\mathscr{F}(L)$ denote the set comprising all finite-dimensional subspaces of $L$ and note that this set is naturally directed by inclusion. To each $M \in \mathscr{F}(L)$ we associate the (orthogonal) projection $P_{M}: L \rightarrow L$ of $L$ onto $M$ along the orthocomplement $M^{\perp}$. When $M \in \mathscr{F}(L)$ and when $\Lambda: L \rightarrow \mathbb{R}$ is a $d$-homogeneous polynomial, the compression $\Lambda_{M}=\Lambda \circ P_{M}$ is again a $d$-homogeneous polynomial. We say that the $d$-homogeneous polynomial $\Lambda: L \rightarrow \mathbb{R}$ is tame when $\Lambda=\Lambda_{M}$ for some $M \in \mathscr{F}(L)$ on which $\Lambda$ is said to be based. Denote by $P^{d} L$ the vector space comprising all tame $d$-homogeneous polynomials on $L$ and by $P_{M}^{d} L$ its subspace comprising all those elements that are based on $M \in \mathscr{F}(L)$.

To each $u \in L$ we associate the linear functional $\phi_{u}: L \rightarrow \mathbb{R}$ defined by the rule that if $z \in L$ then $\phi_{u}(z)=(u \mid z)$. Of course, if $z_{1}, \cdots, z_{d} \in L$ then the product $\phi_{z_{1}}$ $\cdots \phi_{z_{d}}$ lies in $P^{d} L$ : in fact, $P^{d} L$ is the linear span of all such products; indeed, $P^{d} L$ is the linear span of $\left\{\phi^{d}{ }_{z}: z \in L\right\}$ by polarization.

Theorem 1.1. $P^{d} L$ carries a canonical inner product $(\cdot \mid \cdot)$ uniquely determined by the rule that if $x_{1}, \cdots, x_{d}, y_{1}, \cdots y_{d} \in L$ then

$$
\left(\phi_{x_{1}} \cdots \phi_{x_{d}} \mid \phi_{y_{1}} \cdots \phi_{y_{d}}\right)=\sum_{\pi} \prod_{j=1}^{d}\left(x_{j} \mid y_{\pi j}\right)
$$

where $\pi$ runs over the group $S_{d}$ comprising all permutations of $\{1, \cdots, d\}$.

In particular, note that the induced norm $\|\cdot\|$ on $P^{d} L$ has the property that if 
$z_{1}, \cdots, z_{d} \in L$ then

$$
\left\|\phi_{z_{1}} \cdots \phi_{z_{d}}\right\| \leqslant \sqrt{d !}\left\|z_{1}\right\| \cdots\left\|z_{d}\right\| .
$$

Let $\mathbb{N}^{m, d}$ denote the set of all $d$-tuples $D=\left(d_{1}, \cdots, d_{m}\right) \in \mathbb{N}^{m}$ having total degree $|D|=d_{1}+\cdots+d_{m}$ equal to $d$ and write $D !=d_{1} ! \cdots d_{m} !$.

Theorem 1.2. If $M \in \mathscr{F}(L)$ has $\left(u_{1}, \cdots, u_{m}\right)$ as an arthonormal basis then $P_{M}^{d} L$ has $\left(\Phi^{D}: D \in \mathbb{N}^{m, d}\right)$ as an orthonormal basis, where if $D \in \mathbb{N}^{m, d}$ then

$$
\sqrt{D !} \Phi^{D}=\phi_{u_{1}}^{d_{1} \cdots} \phi_{u_{m}}^{d_{m}}
$$

Proof. That the vectors $\left(\Phi^{D}: D \in \mathbb{N}^{m, d}\right)$ span $P_{M}^{d} L$ is at once clear; that they are orthonormal follows upon direct calculation from the formula for the inner product on $P^{d} L$.

With this notation, if $\Lambda \in P_{M}^{d} L$ then Fourier decomposition yields

$$
\Lambda=\sum_{D}\left(\Phi^{D} \mid \Lambda\right) \Phi^{D}
$$

and the Parseval equality yields

$$
\|\Lambda\|^{2}=\sum_{D}\left(\Phi^{D} \mid \Lambda\right)^{2} .
$$

Now, let $N \subset M \in \mathscr{F}(L)$ and note that $P_{N}^{d} L \subset P_{M}^{d} L$ since $P_{N} P_{M}=P_{N}$. Extend an orthonormal basis $\left(u_{1}, \cdots, u_{n}\right)$ for $N$ to an orthonormal basis $\left(u_{1}, \cdots, u_{m}\right)$ for $M$. Embed $\mathbb{N}^{n, d}$ in $\mathbb{N}^{m, d}$ by appending zeros, so that $\mathbb{N}^{n, d}$ is identified with $\left\{D \in \mathbb{N}^{m, d}\right.$ : $\left.n<j \leqslant m \Rightarrow d_{j}=0\right\}$. With this understanding, if $D \in \mathbb{N}^{m, d}$ then

$$
\Phi^{D} \circ P_{N}= \begin{cases}\Phi^{D} & D \in \mathbb{N}^{n, d} \\ 0 & D \notin \mathbb{N}^{n, d} .\end{cases}
$$

Accordingly, orthogonal projection of $P_{M}^{d} L$ onto $P_{N}^{d} L$ is precisely the compression map

$$
P_{M}^{d} L \rightarrow P_{M}^{d} L: \Lambda \mapsto \Lambda \circ P_{N}
$$

Theorem 1.3. Let $N \subset M \in \mathscr{F}(L)$. If $\Lambda: L \rightarrow \mathbb{R}$ is a d-homogeneous polynomial then

$$
\left\|\Lambda_{M}-\Lambda_{N}\right\|^{2}+\left\|\Lambda_{N}\right\|^{2}=\left\|\Lambda_{M}\right\|^{2}
$$

Proof. Merely note that the orthogonal projection of $\Lambda_{M}$ in $P_{N}^{d} L$ is $\Lambda_{M} \circ P_{N}=$ 
$\Lambda_{N}$ and apply the Pythagorean rule.

Introducing the notion central to this, section, we declare the $d$-homogeneous polynomial $\Lambda: L \rightarrow \mathbb{R}$ to be Hilbert-Schmidt when the net $\left(\Lambda_{M}\right.$ : $M \in \mathscr{F}(L))$ in $P^{d} L$ has bounded norms and define

$$
\|\Lambda\|=\sup _{M}\left\|\Lambda_{M}\right\|
$$

Theorem 1.3 tells us that the net $\left(\left\|\Lambda_{M}\right\|: M \in \mathscr{F}(L)\right)$ is increasing, whence also

$$
\|\Lambda\|=\lim _{M}\left\|\Lambda_{M}\right\|
$$

We remark that our notation is consistent, for let $N \in \mathscr{F}(L)$ and let $\Lambda=\Lambda_{N} \in P_{N}^{d} L$ be tame: if also $M \in \mathscr{F}(L)$ then Theorem 1.3 implies that $\left\|\Lambda_{M}\right\| \leqslant\left\|\Lambda_{M+N}\right\|=\left\|\Lambda_{N}\right\|$ so that $\sup \left\{\left\|\Lambda_{M}\right\|: M \in \mathscr{F}(L)\right\}=\left\|\Lambda_{N}\right\|$. Denote by $\mathbb{P}^{d} L$ the set comprising all Hilbert-Schmidt $d$-homogeneous polynomials on $L$. It is at once clear that $\mathbb{P}^{d} L$ is a vector space on which $\|\cdot\|$ is a norm.

In fact, the norm $\|\cdot\|$ on $\mathbb{P}^{d} L$ is defined by an inner product: if $\Lambda^{\prime}, \Lambda^{\prime \prime} \in \mathbb{P}^{d} L$ and if $M \in \mathscr{F}(L)$ then

$$
\left\|\left(\Lambda^{\prime}+\Lambda^{\prime \prime}\right)_{M}\right\|^{2}+\left\|\left(\Lambda^{\prime}-\Lambda^{\prime \prime}\right)_{M}\right\|^{2}=2\left(\left\|\Lambda_{M}^{\prime}\right\|^{2}+\left\|\Lambda_{M}^{\prime \prime}\right\|^{2}\right)
$$

whence passage to the limit as $M \rightarrow L$ yields

$$
\left\|\Lambda^{\prime}+\Lambda^{\prime \prime}\right\|^{2}+\left\|\Lambda^{\prime}-\Lambda^{\prime \prime}\right\|^{2}=2\left(\left\|\Lambda^{\prime}\right\|^{2}+\left\|\Lambda^{\prime \prime}\right\|^{2}\right) .
$$

Thus $\|\cdot\|$ satisfies the parallelogram law and so arises from an inner product $(\cdot \mid \cdot)$ given by

$$
\Lambda^{\prime}, \Lambda^{\prime \prime} \in \mathbb{P}^{d} L \Rightarrow\left(\Lambda^{\prime} \mid \Lambda^{\prime \prime}\right)=\frac{1}{4}\left(\left\|\Lambda^{\prime}+\Lambda^{\prime \prime}\right\|^{2}-\left\|\Lambda^{\prime}-\Lambda^{\prime \prime}\right\|^{2}\right) .
$$

As remarked above, the inner product space $P^{d} L$ is included isometrically in the inner product space $\mathbb{P}^{d} L$.

As is almost obvious by construction, we claim that $P^{d} L$ is dense in $\mathbb{P}^{d} L$. To see this and more, note first that if $\Lambda \in \mathbb{P}^{d} L$ and $N \in \mathscr{F}(L)$ then multiple applications of Theorem 1.3 imply

$$
\begin{aligned}
\left\|\Lambda-\Lambda_{N}\right\|^{2} & =\sup _{M}\left\|\left(\Lambda-\Lambda_{N}\right)_{M}\right\|^{2} \\
& =\sup _{M \supset N}\left\|\left(\Lambda-\Lambda_{N}\right)_{M}\right\|^{2} \\
& =\sup _{M \supset N}\left\|\Lambda_{M}\right\|^{2}-\left\|\Lambda_{N}\right\|^{2} \\
& =\|\Lambda\|^{2}-\left\|\Lambda_{N}\right\|^{2}
\end{aligned}
$$

whence Theorem 1.3 extends to the assertion that

$$
\|\Lambda\|^{2}=\left\|\Lambda-\Lambda_{N}\right\|^{2}+\left\|\Lambda_{N}\right\|^{2} .
$$


Theorem 1.4. If $\Lambda \in \mathbb{P}^{d} L$ then the net $\left(\Lambda_{M}: M \in \mathscr{F}(L)\right)$ converges to $\Lambda$ in $\mathbb{P}^{d} L$.

Proof. Given $\varepsilon>0$ choose $M_{\varepsilon} \in \mathscr{F}(L)$ so that $\left\|\Lambda_{M_{\varepsilon}}\right\|^{2} \geqslant\|\Lambda\|^{2}-\varepsilon^{2}$. Now, if $M_{\varepsilon} \subset M$ $\in \mathscr{F}(L)$ then $\left\|\Lambda_{M_{\varepsilon}}\right\| \leqslant\left\|\Lambda_{M}\right\|$ by Theorem 1.3 and therefore $\left\|\Lambda-\Lambda_{M}\right\| \leqslant \varepsilon$ by the extended version just established.

It is convenient that the functional values of polynomials in $\mathbb{P}^{d} L$ may be recovered by taking inner products.

Theorem 1.5. If $\Lambda \in \mathbb{P}^{d} L$ and $z \in L$ then

$$
d ! \Lambda(z)=\left(\phi_{z}^{d} \mid \Lambda\right)
$$

Proof. If $\Lambda \in P^{d} L$ then the asserted equality holds by linearity and the formula for the inner product on $P^{d} L$. If $\Lambda \in \mathbb{P}^{d} L$ and if $M \in \mathscr{F}(L)$ contains $z$ then

$$
d ! \Lambda(z)=d ! \Lambda_{M}(z)=\left(\phi_{z}^{d} \mid \Lambda_{M}\right)
$$

whence an application of Theorem 1.4 shows that passage to the limit as $M \rightarrow L$ yields the asserted equality in general.

The principle of polarization extends this to the result that if $\Lambda \in \mathbb{P}^{d} L$ and $z_{1}, \cdots, z_{d} \in L$ then

$$
d ! \underline{\Lambda}\left(z_{1}, \cdots, z_{d}\right)=\left(\phi_{z_{1}} \cdots \phi_{z_{d}} \mid \Lambda\right) .
$$

From this, it follows at once that the symmetric $d$-form $\underline{\Lambda}$ is bounded: in fact,

$$
\left|\underline{\Lambda}\left(z_{1}, \cdots, z_{d}\right)\right| \leqslant \frac{\|\Lambda\|}{\sqrt{d !}}\left\|z_{1}\right\| \cdots\left\|z_{d}\right\| .
$$

Not only does $\mathbb{P}^{d} L$ contain $P^{d} L$ as a dense subspace: $\mathbb{P}^{d} L$ is actually the completion of $P^{d} L$.

Theorem 1.6. $\quad \mathbb{P}^{d} L$ is a Hilbert space.

Proof. Let $\left(\Lambda^{n}: n \in \mathbb{N}\right)$ be a Cauchy sequence in $\mathbb{P}^{d} \boldsymbol{L}$. The inequality established after Theorem 1.5 shows that if $z_{1}, \cdots, z_{d} \in \mathcal{L}$ then the sequence $\left(\Lambda^{n}\left(z_{1}, \cdots, z_{d}\right): n \in \mathbb{N}\right)$ is Cauchy, so we may put

$$
\underline{\Lambda}\left(z_{1}, \cdots, z_{d}\right)=\lim _{n} \Lambda^{n}\left(z_{1}, \cdots, z_{d}\right)
$$

and thereby define a symmetric $d$-form $\Lambda$ on $L$. Let $\varepsilon>0$ and choose $n_{\varepsilon} \in \mathbb{N}$ so that if $p, q \geqslant n_{\varepsilon}$ then $\left\|\Lambda^{p}-\Lambda^{q}\right\| \leqslant \varepsilon$. If $M \in \mathscr{F}(L)$ then the discussion of Theorem 
1.2 enables us to infer from

$$
\underline{\Lambda_{M}^{j}}\left(z_{1}, \cdots, z_{d}\right)=\underline{\Lambda^{j}}\left(P_{M} z_{1}, \cdots, P_{M} z_{d}\right) \rightarrow \underline{\Lambda}\left(P_{M} z_{1}, \cdots, P_{M} z_{d}\right)=\underline{\Lambda_{M}}\left(z_{1}, \cdots, z_{d}\right)
$$

that

$$
\Lambda_{M}^{j}=\sum_{D}\left(\Phi^{D} \mid \Lambda_{M}^{j}\right) \Phi^{D} \rightarrow \sum_{D}\left(\Phi^{D} \mid \Lambda_{M}\right) \Phi^{D}=\Lambda_{M}
$$

whence if $n \geqslant n$ then

$$
\left\|\left(\Lambda-\Lambda^{n}\right)_{M}\right\|=\lim _{j}\left\|\Lambda_{M}^{j}-\Lambda_{M}^{n}\right\| \leqslant \varepsilon
$$

From this, it follows both that $\Lambda \in \mathbb{P}^{d} L$ and that $\Lambda^{n} \rightarrow \Lambda$ in $\mathbb{P}^{d} L$.

We may exhibit an orthonormal basis for the Hilbert space $\mathbb{P}^{d} L$ as follows. For $\mathscr{A}$ any set, let $\mathbb{N}^{\mathscr{A}, d}$ denote the set comprising all maps $D: \mathscr{A} \rightarrow \mathbb{N}$ with total degree

$$
\sum_{a \in \mathscr{A}} D(a)=d
$$

and write

$$
\prod_{a \in \mathscr{A}}\{D(a) !\}=D !
$$

where the sum and product are finite of course.

Theorem 1.7. If $L$ has $\left(u_{a}: a \in \mathscr{A}\right)$ as an orthonormal basis then $\mathbb{P}^{d} L$ has $\left(\Phi^{D}: D \in \mathbb{N}^{A, d}\right)$ as an orthonormal basis, where if $D \in \mathbb{N}^{A, d}$ then

$$
\sqrt{D !} \Phi^{D}=\prod_{a \in \mathscr{A}} \phi_{u_{a}}^{D(a)} .
$$

Proof. Orthonormality of the displayed vectors in $P^{d} L$ follows by direct calculation as for Theorem 1.2. To verify completeness of the system, let $\Lambda \in$ $\mathbb{P}^{d} L$ and assume that if $D \in \mathbb{N}^{\mathscr{A} \cdot d}$ then $\left(\Phi^{D} \mid \Lambda\right)=0$. From this assumption, the equality after Theorem 1.5 implies that $\Lambda\left(z_{1}, \cdots, z_{d}\right)=0$ when $z_{1}, \cdots, z_{d}$ lie in the linear span of $\left(u_{a}: a \in \mathscr{A}\right)$ and then the inequality after Theorem 1.5 implies that $\Lambda\left(z_{1}, \cdots, z_{d}\right)=0$ when $z_{1}, \cdots, z_{d} \in L$ are arbitrary.

In particular, each $\Lambda \in \mathbb{P}^{d} L$ has a generalized Fourier decomposition

$$
\Lambda=\sum_{D}\left(\Phi^{D} \mid \Lambda\right) \Phi^{D}
$$


for which the Parseval equality reads

$$
\|\Lambda\|^{2}=\sum_{D}\left(\Phi^{D} \mid \Lambda\right)^{2}
$$

Incidentally, we are now able to show that our notion of Hilbert-Schmidt polynomial agrees with the standard one. Too each $A=\left(a_{1}, \cdots, a_{d}\right) \in \mathscr{A}^{d}$ we associate $D_{A} \in \mathbb{N}^{\mathscr{A l}, d}$ by requiring that each $a \in \mathscr{A}$ appears $D_{A}(a)$ times in the list $\left(a_{1}, \cdots, a_{d}\right)$. The resulting map $\mathscr{A}^{d} \rightarrow \mathbb{N}^{d, d}$ is surjective, the preimage of $D \in \mathbb{N}^{d, d}$ having $d ! / D$ ! as its cardinality.

Theorem 1.8. If $\Lambda \in \mathbb{P}^{d} L$ and if $\left(u_{a}: a \in \mathscr{A}\right)$ is an orthonormal basis for $L$ then

$$
\|\Lambda\|^{2}=d ! \sum_{A}\left|\underline{\Lambda}\left(u_{a_{1}}, \cdots, u_{a_{d}}\right)\right|^{2} .
$$

Proof. If $A=\left(a_{1}, \cdots, a_{d}\right) \in \mathscr{A}^{d}$ then Theorem 1.5 yields

$$
d ! \Lambda\left(u_{a_{1}}, \cdots, u_{a_{d}}\right)=\sqrt{D_{A} !}\left(\Phi^{D_{A}} \mid \Lambda\right)
$$

whence it follows that

$$
d ! \sum_{A}\left|\Lambda\left(u_{a_{1}}, \cdots, u_{a_{d}}\right)\right|^{2}=\sum_{A} \frac{D_{A} !}{d !}\left(\Phi^{D_{A}} \mid \Lambda\right)^{2}=\sum_{D} \frac{D !}{d !} \sum_{D_{A}=D}\left(\Phi^{D_{A}} \mid \Lambda\right)^{2}=\sum_{D}\left(\Phi^{D} \mid \Lambda\right)^{2}
$$

by summation over the fibres of $\mathscr{A}^{d} \rightarrow \mathbb{N}^{A d, d}$ as an intermediate step.

Thus far, we have considered only polynomials possessing a fixed homogeneous degree. More generally, by a polynomial on $L$ we mean a finite sum of homogeneous polynomials on $L$. When $\Lambda: L \rightarrow \mathbb{R}$ is a polynomial we shall denote by ${ }^{d} \Lambda$ its degree $d$ homogeneous component, whence $\Lambda$ decomposes as the finite sum

$$
\Lambda=\sum_{d \geqslant 0}^{d} \Lambda
$$

If $\Lambda: L \rightarrow \mathbb{R}$ is a polynomial and if $M \in \mathscr{F}(L)$ then the compression $\Lambda_{M}=\Lambda \circ P_{M}$ is again a polynomial, said to be tame and based on $M$. The space $P L$ of tame polynomials on $L$ is thus the algebraic direct sum

$$
P L=\underset{d \geqslant 0}{\bigoplus} P^{d} L
$$

which we equip with the inner product that renders the decomposition orthogonal and restricts to the original on each summand. We say that the polynomial $\Lambda: L \rightarrow \mathbb{R}$ is Hilbert-Schmidt when the net $\left(\Lambda_{M}: M \in \mathscr{F}(L)\right)$ in $P L$ has bounded norms and write 


$$
\|\Lambda\|=\sup _{M}\left\|\Lambda_{M}\right\|=\lim _{M}\left\|\Lambda_{M}\right\|
$$

The space $\mathbb{P} L$ of all Hilbert-Schmidt polynomials on $L$ is the algebraic direct sum

$$
\mathbb{P} L=\underset{d \geqslant 0}{\bigoplus \mathbb{P}^{d} L}
$$

which we provide with the inner product that renders the decomposition orthogonal and restricts to the original on each summand.

We close this section by discussing briefly the effect of translations on polynomials. Thus, to each $u \in L$ and each polynomial $\Lambda: L \rightarrow \mathbb{R}$ we associate polynomials $T^{u} \Lambda$ and $\Lambda^{u}$ on $L$ defined by

$$
T^{u} \Lambda: L \rightarrow \mathbb{R}: z \mapsto \Lambda(u+z)
$$

and

$$
\Lambda^{u}=T^{u} \Lambda-\Lambda
$$

We remark that if $\Lambda$ is $(d+1)$-homogeneous then

$$
\Lambda^{u}=\sum_{j=0}^{d} \Lambda^{u}
$$

where if $0 \leqslant j \leqslant d$ then ${ }^{j} \Lambda^{u}$ is the $j$-homogeneous polynomial given by the rule that if $z_{1}, \cdots, z_{j} \in L$ then

$$
{ }^{j} \underline{\Lambda}^{u}\left(z_{1}, \cdots, z_{j}\right)=\left(\begin{array}{c}
d+1 \\
j
\end{array}\right) \underline{\Lambda}\left(u, \cdots, u, z_{1}, \cdots, z_{j}\right)
$$

with $d+1-j$ terms $u$ on the right. We also remark that in this case, if ${ }^{d} \Lambda^{u}$ is Hilbert-Schmidt then ${ }^{j} \Lambda^{u}$ is Hilbert-Schmidt whenever $0 \leqslant j \leqslant d$.

Theorem 1.9. Let $\Lambda$ be a $(d+1)$-homogeneous polynomial on $L$ with $d>0$. If $\Lambda^{u} \in \mathbb{P} L$ whenever $u \in L$ then $\Lambda$ is bounded.

Proof. We claim that the linear map

$$
L \rightarrow \mathbb{P}^{d} L: \imath \mapsto^{d} \Lambda^{u}
$$

is continuous, by application of the closed graph theorem. Thus, let $\left(u_{n}: n \in \mathbb{N}\right)$ be a null sequence in $L$ and let ${ }^{d} \Lambda^{u_{n}} \rightarrow \Lambda^{\prime}$ in $\mathbb{P}^{d} L$. If $z_{1}, \cdots, z_{d} \in L$ then the equality after Theorem 1.5 yields

$$
\begin{aligned}
\left(\phi_{z_{1}} \cdots \phi_{z_{d}}{ }^{d} \Lambda^{u_{n}}\right) & =d !^{d} \Lambda^{\Lambda_{n}}\left(z_{1}, \cdots, z_{d}\right) \\
& =(d+1) ! \Lambda\left(u_{n}, z_{1}, \cdots, z_{d}\right) \\
& =\left(\phi_{z_{1}} \cdots \phi_{z_{d}} \phi_{u_{n}} \mid \Lambda\right)
\end{aligned}
$$


whence the inequality after Theorem 1.5 yields

$$
\left|\left(\left.\phi_{z_{1}} \cdots \phi_{z_{d}}\right|^{d} \Lambda^{u_{n}}\right)\right| \leqslant \sqrt{(d+1) !}\|\Lambda\|\left\|z_{1}\right\| \cdots\left\|z_{d}\right\|\left\|u_{n}\right\|
$$

and passage to the limit as $n \rightarrow \infty$ yields

$$
d ! \Lambda^{\prime}\left(z_{1}, \cdots, z_{d}\right)=\left(\phi_{z_{1}} \cdots \phi_{z_{d}} \mid \Lambda^{\prime}\right)=0 .
$$

Now, let $K \geqslant 0$ be such that $\left\|{ }^{d} \Lambda^{u}\right\| \leq K\|u\|$ whenever $u \in L$. If $z_{0}, z_{1}, \cdots, z_{d} \in L$ then

$$
\left|\underline{\Lambda}\left(z_{0}, z_{1}, \cdots, z_{d}\right)\right|=\left.\frac{1}{(d+1) !}\right|^{d} \underline{\Lambda}^{z_{0}}\left(z_{1}, \cdots, z_{d}\right) \mid \leqslant \frac{K}{(d+1) \sqrt{d !}}\left\|z_{0}\right\|\left\|z_{1}\right\| \cdots\left\|z_{d}\right\|
$$

so that $\Lambda$ is bounded as claimed.

By way of definition, we shall say that the polynomial $\Lambda: L \rightarrow \mathbb{R}$ is differentially Hilbert-Schmidt when the difference $\Lambda^{u}=T^{u} \Lambda-\Lambda$ is HilbertSchmidt for every $u \in L$.

\section{Wick-Ordered Polynomials}

In principle, the Wick ordering of Hilbert-Schmidt polynomials amounts to their orthogonalization relative to a Gaussian distribution on Hilbert space. To explain this in detail, we begin by recalling basic facts pertaining to Hermite polynomials and presenting a very brief account of $Q$-space.

Fix a positive number $\sigma$ and let $\mu$ denote the Gaussian probability measure on $\mathbb{R}$ having density function

$$
\mathbb{R} \rightarrow \mathbb{R}: t \mapsto \frac{1}{\sigma \sqrt{2 \pi}} \exp \left[-\frac{t^{2}}{2 \sigma^{2}}\right]
$$

relative to normalized Lebesgue measure, so that $\mu$ is the law of a normal random variable having variance $\sigma^{2}$ and mean zero. The Hermite polynomials $H^{n}(s)$ are the monic polynomials that arise when the powers $s^{n}$ are orthogonalized relative to the weight $\mu$. Explicitly, if $n \in \mathbb{N}$ then the Hermite polynomial $H^{n}: \mathbb{R} \rightarrow \mathbb{R}$ has value at $s \in \mathbb{R}$ given by

$$
H^{n}(s)=\left.\left(-\sigma^{2}\right)^{n} e^{\frac{s^{2}}{2 \sigma^{2}}} \frac{d^{n}}{d t^{n}}\left\{e^{-\frac{t^{2}}{2 \sigma^{2}}}\right\}\right|_{t=s}
$$

For our purposes, the following equivalent definition and fundamental properties will be particularly important.

Theorem 2.1. If $n \in \mathbb{N}$ and $s \in \mathbb{R}$ then

$$
H^{n}(s)=\int_{\mathbb{R}}(s+i t)^{n} d \mu(t)
$$

and 


$$
\int_{\mathbb{R}} H^{n} H^{n} d \mu=n ! \sigma^{2 n}
$$

while if $p, q \in \mathbb{N}$ are distinct then

$$
\int_{\mathbb{R}} H^{p} H^{q} d \mu=0
$$

We should mention that the literature contains different conventions regarding Hermite polynomials. Thus, Hermite polynomials are sometimes normalized so as to have unit norm rather than to be monic. Also, the variance $\sigma^{2}$ of the Gaussian is usually assigned a specific value: typically, either $\sigma^{2}=1$ or $\sigma^{2}=\frac{1}{2}$

To construct $Q$-space, we proceed as follows. Each finite-dimensional subspace $M \in \mathscr{F}(L)$ of $L$ is equipped with the Gaussian probability measure $\mu$ having density function

$$
M \rightarrow \mathbb{R}: z \mapsto\left(2 \pi \sigma^{2}\right)^{-\frac{1}{2} \operatorname{dim} M} \exp \left[-\frac{\|z\|^{2}}{2 \sigma^{2}}\right]
$$

relative to normalized Lebesgue measure. Let $H(M)$ stand for the set comprising all functions $f: M \rightarrow \mathbb{C}$ based on $M$ in the sense that $f=f_{M} \circ P_{M}$ for some continuous function $f_{M}: M \rightarrow \mathbb{C}$ square-integrable relative to $\mu$. Elements of the complex vector space

$$
H(L)=\cup\{H(M): M \in \mathscr{F}(L)\}
$$

will be called tame functions on $L$. The Gaussian probability measures on the elements of $\mathscr{F}(L)$ being consistent, an inner product $\langle\cdot \mid \cdot\rangle$ on $H(L)$ is well defined by the rule that if $f$ and $g$ lie in $H(L)$ then

$$
\langle f \mid g\rangle=\int_{M} \overline{f g} d \mu
$$

for any $M \in \mathscr{F}(L)$ on which both $f$ and $g$ are based. The resulting complex Hilbert space completion of $H(L)$ is precisely $Q$-space $\mathbb{H}(L)$ over $L$.

Plainly, if $M \in \mathscr{F}(L)$ then $H(M)$ contains each function $f=f_{M} \circ P_{M}$ for which $f_{M}: M \rightarrow \mathbb{C}$ is a polynomial. Accordingly, the vector space $P L$ of all tame polynomials on $L$ is contained in $H(L)$ and thereby contained in $\mathbb{H}(L)$.

Note that each real polynomial $\Lambda: L \rightarrow \mathbb{R}$ naturally extends to a complex polynomial $\Lambda: L^{\mathbb{C}} \rightarrow \mathbb{C}$ on the complexification $L^{\mathbb{C}}$ of $L:$ in case $\Lambda$ is $d$-homogeneous, the associated complex symmetric $d$-form $\Lambda: L^{\mathbb{C}} \times \cdots \times L^{\mathbb{C}} \rightarrow \mathbb{C}$ is determined by complex multilinearity.

Now, if $\Lambda \in P L$ is a tame polynomial on $L$ then we define $\Gamma(\Lambda): L \rightarrow \mathbb{C}$ by the rule that if $z \in L$ then 


$$
\Gamma(\Lambda)(z)=\langle 1 \mid \Lambda(z+i \cdot)\rangle
$$

so that if integration extends over $M \in \mathscr{F}(L)$ on which $\Lambda$ is based then

$$
\Gamma(\Lambda)(z)=\int_{M} \Lambda(z+i w) d \mu(w) .
$$

Theorem 2.2. If $n \in \mathbb{N}$ and if $u \in L$ is a unit vector then

$$
\Gamma\left(\phi_{u}^{n}\right)=H^{n} \circ \phi_{u}
$$

which has norm in $\mathbb{H I}(L)$ given by

$$
\left\|\Gamma\left(\phi_{u}^{n}\right)\right\|^{2}=n ! \sigma^{2 n}
$$

Proof. Direct calculation. If $M \in \mathscr{F}(L)$ is the real line spanned by $u$ and if $z$ $\in L$ then

$$
\begin{aligned}
\Gamma\left(\phi_{u}^{n}\right)(z) & =\int_{M} \phi_{u}(z+i w)^{n} d \mu(w) \\
& =\int_{\mathbb{R}}((u \mid z)+i t)^{n} d \mu(t) \\
& =H^{n}(u \mid z) \\
& =H^{n} \circ \phi_{u}(z) .
\end{aligned}
$$

The expression for the norm of $\Gamma\left(\phi_{u}^{n}\right)$ in $\mathbb{H I}(L)$ comes straight from Theorem 2.1 .

We remark that as $H^{n}$ is a degree $n$ monic real polynomial, so the difference $\Gamma\left(\phi_{u}^{n}\right)-\phi_{u}^{n}$ is a (real) polynomial based on the line spanned by $u$ and has degree strictly less than $n$.

Theorem 2.3. If $\Lambda \in P_{M}^{d} L$ for some $M \in \mathscr{F}(L)$ then $\Gamma(\Lambda) \in P L$ is based on $M$ and $\Gamma(\Lambda)-\Lambda$ has degree strictly less than $d$.

Proof. By linearity, it is enough to let $M$ have $\left(u_{1}, \cdots, u_{m}\right)$ as orthonormal basis, to let $D=\left(d_{1}, \cdots, d_{m}\right) \in \mathbb{N}^{m, d}$ and to consider $\Lambda=\Phi^{D}$ where

$$
\sqrt{D !} \Phi^{D}=\phi_{u_{1}}^{d_{1} \cdots} \phi_{u_{m}}^{d_{m}}
$$

For this, Theorem 2.2 and orthogonality of the lines spanned by $u_{1}, \cdots, u_{m}$ together imply that

$$
\begin{aligned}
\sqrt{D !} \Gamma\left(\Phi^{D}\right) & =\Gamma\left(\phi_{u_{1}}^{d_{1}}\right) \cdots \Gamma\left(\phi_{u_{m}}^{d_{m}}\right) \\
& =\left(H^{d_{1} \circ} \phi_{u_{1}}\right) \cdots\left(H^{d_{m}} \circ \phi_{u_{m}}\right) .
\end{aligned}
$$

Expansion on the remark after Theorem 2.2 concludes the proof. 
Further to the proof, notice that Theorem 2.2 and orthogonality of the lines spanned by $u_{1},{ }^{\cdots}, u_{m}$ also imply that the norm of $\Gamma\left(\Phi^{D}\right)$ in $\mathbb{H}(L)$ is given by

$$
\left\|\Gamma\left(\Phi^{D}\right)\right\|=\sigma^{d} .
$$

Theorem 2.4. The map $\Gamma: P L \rightarrow P L$ is a linear automorphism, its inverse being given by the rule that if $\Lambda \in P L$ is based on $M \in \mathscr{F}(L)$ and if $z \in L$ then

$$
\left(\Gamma^{-1} \Lambda\right)(z)=\int_{M} \Lambda(z+w) d \mu(w) .
$$

Proof. Let $\Gamma^{\prime}$ temporarily denote the putative inverse for $\Gamma$ defined by the displayed formula. Let $n \in \mathbb{N}$, let $u \in L$ be a unit vector spanning the line $M_{0}$ and let $z \in L$. From

$$
\begin{aligned}
\Gamma\left(\phi_{u}^{n}\right)(z) & =\int_{M_{0}} \phi_{u}(z+w)^{n} d \mu(w) \\
& =\int_{\mathbb{R}}((u \mid z)+t)^{n} d \mu(t)
\end{aligned}
$$

it is plain that $\Gamma^{\prime}\left(\phi_{u}^{n}\right)$ is a degree $n$ polynomial based on $M_{0}$. From the readily verified formula

$$
s \in \mathbb{R} \Rightarrow \int_{\mathbb{R}} H^{n}(s+t) d \mu(t)=s^{n}
$$

it is evident that

$$
\begin{aligned}
\Gamma^{\prime} \Gamma\left(\phi_{u}^{n}\right)(z) & =\int_{\mathbb{R}} H^{n}((u \mid z)+t) d \mu(t) \\
& =\phi_{u}^{n}(z)
\end{aligned}
$$

Now, if $M \in \mathscr{F}(L)$ has orthonormal basis $\left(u_{1}, \cdots, u_{m}\right)$ and if $D \in \mathbb{N}^{m, d}$ then factorization shows that $\Gamma^{\prime}\left(\Phi^{D}\right)$ is a degree $d$ polynomial based on $M$ and that $\Gamma^{\prime} \Gamma\left(\Phi^{D}\right)=\Phi^{D}$. By linearity, it follows that $\Gamma^{\prime}$ maps $P L$ to itself and has the property that $\Gamma^{\prime} \circ \Gamma$ is the identity. Finally, we claim that $\Gamma^{\prime} \circ \Gamma=\Gamma \circ \Gamma^{\prime}$ : if $\Lambda \in$ $P L$ is based on $M \in \mathscr{F}(L)$ and if $z \in L$ then

$$
\begin{aligned}
\left(\Gamma^{\prime} \Gamma \Lambda\right)(z) & =\int_{M} \Gamma \Lambda(z+x) d \mu(x) \\
& =\int_{M} \int_{M} \Lambda(z+x+i y) d \mu(y) d \mu(x) \\
& =\int_{M} \int_{M} \Lambda(z+i y+x) d \mu(x) d \mu(y) \\
& =\int_{M} \Gamma^{\prime} \Lambda(z+i y) d \mu(y) \\
& =\left(\Gamma \Gamma^{\prime} \Lambda\right)(z) .
\end{aligned}
$$

We are more interested in $\Gamma$ as a linear map from $P L$ to $\mathbb{H}(L)$. As such, its 
rôle is to orthogonalize relative to the Gaussian distribution $\mu$.

Theorem 2.5. The linear map $\Gamma: P L \rightarrow \mathbb{H}(L)$ has the following properties.

(i) If $\Lambda^{\prime}, \Lambda^{\prime \prime} \in P^{d} L$ then

$$
\left\langle\Gamma\left(\Lambda^{\prime}\right) \mid \Gamma\left(\Lambda^{\prime \prime}\right)\right\rangle=\sigma^{2 d}\left(\Lambda^{\prime} \mid \Lambda^{\prime \prime}\right) .
$$

(ii) If $\Lambda^{\prime} \in P^{d^{\prime}} L$ and $\Lambda^{\prime \prime} \in P^{d^{\prime \prime}} L$ where $d^{\prime}$ and $d^{\prime \prime}$ are distinct then

$$
\left\langle\Gamma\left(\Lambda^{\prime}\right) \mid \Gamma\left(\Lambda^{\prime \prime}\right)\right\rangle=0 .
$$

Proof. If $\Lambda^{\prime}, \Lambda^{\prime \prime} \in P L$ are based on $M^{\prime}, M^{\prime \prime} \in \mathscr{F}(L)$ then both $\Lambda^{\prime}$ and $\Lambda^{\prime \prime}$ are based on $M=M^{\prime}+M^{\prime \prime}$. Let $M$ have $\left(u_{1}, \cdots, u_{m}\right)$ as orthonormal basis. If $D \in \mathbb{N}^{m, d}$ then

$$
\left\langle\Gamma\left(\Phi^{D}\right) \mid \Gamma\left(\Phi^{D}\right)\right\rangle=\sigma^{2 d}\left(\Phi^{D} \mid \Phi^{D}\right)
$$

as noticed after Theorem 2.3. It follows similarly from Theorem 2.1 and orthogonality of the lines spanned by $u_{1}, \cdots, u_{m}$ that if $D^{\prime} \in \mathbb{N}^{m, d^{\prime}}$ and $D^{\prime \prime} \in \mathbb{N}^{m, d^{\prime \prime}}$ are distinct then

$$
\left\langle\Gamma\left(\Phi^{D^{\prime}}\right) \mid \Gamma\left(\Phi^{D^{\prime \prime}}\right)\right\rangle=0
$$

whether or not $d^{\prime}$ and $d^{\prime \prime}$ are distinct. All that remains is to invoke bilinearity.

In particular, each restriction $\Gamma: P^{d} L \rightarrow \mathbb{H}(L)$ is isometric up to a scalar factor:

$$
\Lambda \in P^{d} L \Rightarrow\|\Gamma(\Lambda)\|=\sigma^{d}\|\Lambda\|
$$

where the norms on right and left are calculated in $P^{d} L \subset P L$ and $\mathbb{H}(L)$ respectively. It is perhaps worth remarking that the scalar factor $\sigma^{d}$ may be absorbed by rescaling the inner product on $P^{d} L$.

Of course, extension by continuity yields a linear map $\Gamma: \mathbb{P} L \rightarrow \mathbb{H I}(L)$ having properties analogous to those of the original: if $\Lambda^{\prime}, \Lambda^{\prime \prime} \in \mathbb{P}^{d} L$ then

$$
\left\langle\Gamma\left(\Lambda^{\prime}\right) \mid \Gamma\left(\Lambda^{\prime \prime}\right)\right\rangle=\sigma^{2 d}\left(\Lambda^{\prime} \mid \Lambda^{\prime \prime}\right)
$$

while if $\Lambda^{\prime} \in \mathbb{P}^{d^{\prime}} L$ and $\Lambda^{\prime \prime} \in \mathbb{P}^{d^{\prime \prime}} L$ where $d^{\prime}$ and $d^{\prime \prime}$ are distinct then

$$
\left\langle\Gamma\left(\Lambda^{\prime}\right) \mid \Gamma\left(\Lambda^{\prime \prime}\right)\right\rangle=0 \text {. }
$$

Indeed, if $\Lambda \in \mathbb{P} L$ then the net $\left(\Lambda_{M}: M \in \mathscr{F}(L)\right)$ in $P L$ converges to $\Lambda$ in $\mathbb{P} L$ thanks to Theorem 1.4 applied to homogeneous components; accordingly, Theorem 2.5 then implies that net $\left(\Gamma\left(\Lambda_{M}\right): M \in \mathscr{F}(L)\right)$ in $P L \subset \mathbb{H}(L)$ is Cauchy and hence converges, its limit in $\mathbb{H}(L)$ being $\Gamma(\Lambda)$ by definition. Thus, if $\Lambda \in \mathbb{P} L$ has homogeneous decomposition 


$$
\Lambda=\sum_{d \geqslant 0}^{d} \Lambda
$$

then $\Gamma(\Lambda) \in \mathbb{H}(L)$ has orthogonal decomposition

$$
\left.\Gamma(\Lambda)=\sum_{d \geqslant 0} \Gamma{ }^{d} \Lambda\right)
$$

and

$$
\|\Gamma(\Lambda)\|^{2}=\sum_{d \geqslant 0} \sigma^{2 d}\|d\|^{2} .
$$

Let $\left(u_{a}: a \in \mathscr{A}\right)$ be an orthonormal basis for $L$ and let $\left(\Phi^{D}: D \in \mathbb{N}^{\mathbb{d}, d}, d \in \mathbb{N}\right)$ be the resulting orthonormal basis for $\mathbb{P} L$ as before. For each $d \in \mathbb{N}$ and each $D$ $\in \mathbb{N}^{\mathscr{A}, d}$ let us define $\Psi^{D} \in P L \subset \mathbb{H}(L)$ by

$$
\sqrt{D !} \Psi^{D}=\prod_{a \in \mathscr{A}} \psi_{u_{a}}^{D(a)}
$$

where if $a \in \mathscr{A}$ then

$$
\phi_{u_{a}}^{D(a)}=H^{D(a)} \circ \phi_{u_{a}} .
$$

In terms of this notation, we have the following expression for $\Gamma$.

Theorem 2.6. If $\Lambda \in \mathbb{P} L$ has the generalized Fourier decomposition

$$
\Lambda=\sum_{d \geqslant 0} \sum_{|D|=d} \lambda_{D} \Phi^{D}
$$

then $\Gamma(\Lambda) \in \mathbb{H}(L)$ has the orthogonal decomposition

$$
\Gamma(\Lambda)=\sum_{d \geqslant 0} \sum_{|D|=d} \lambda_{D} \Psi^{D} .
$$

Proof. Theorem 2.2 and orthogonality of the lines spanned by the vectors $\left(u_{a}: a \in \mathscr{A}\right)$ imply that if $d \in \mathbb{N}$ and $D \in \mathbb{N}^{\mathscr{A}, d}$ then $\Gamma\left(\Phi^{D}\right)=\Psi^{D}$. From this, the asserted equality follows at once by linearity and continuity of $\Gamma$.

In the literature, $\Gamma(\Lambda)$ is traditionally written as : $\Lambda$ : and is obtained from $\Lambda$ by the process of Wick ordering. Accordingly, we refer to $\Gamma$ as the Wick transform; the symbol $\Gamma$ is for Gauss, after whom the transform is also named.

We now show how the Wick transform leads to a Hildert-Schmidt-strong continuous representation on $Q$-space $\mathbb{H}(L)$ of the additive group underlying $\mathbb{P} L$.

First, if $\Lambda \in P L$ is a tame polynomial on $L$ then of course the unitary scalar 
function $\exp \left\{\Gamma(\Lambda) / 2 i \sigma^{2}\right\}$ on $L$ is tame, whence an isometric linear map $U_{\Lambda}$ : $H(L) \rightarrow H(L)$ is defined by prescribing that if $f \in H(L)$ then $U_{A} f \in H(L)$ is the pointwise product

$$
U_{\Lambda} f=\exp \left\{\frac{\Gamma(\Lambda)}{2 i \sigma^{2}}\right\} f
$$

We shall continue to use the same symbol to denote the unique extension of $U_{A}$ to an isometric linear map from $\mathbb{H}(L)$ to itself.

Next, let $\Lambda \in \mathbb{P} L$ be a Hilbert-Schmidt polynomial on $L$. For convenience, when $M \in \mathscr{F}(L)$ let us agree temporarily to write $U_{M}$ in place of $U_{\Lambda_{M}}$. Now, let $f$ $\in H(L)$ be a bounded tame function on $L$ and put sup $|f|=K$. The elementary estimate

$$
t^{\prime}, t^{\prime \prime}=\mathbb{R} \Rightarrow\left|e^{i t^{\prime}}-e^{i t^{\prime \prime}}\right| \leqslant\left|t^{\prime}-t^{\prime \prime}\right|
$$

implies that if $M^{\prime}, M^{\prime \prime} \in \mathscr{F}(L)$ and $M=M^{\prime}+M^{\prime \prime}$ then

$$
\left\|U_{M^{\prime}} f-U_{M^{\prime \prime}} f\right\|^{2} \leqslant \frac{K^{2}}{4 \sigma^{4}} \int_{M}\left|\Gamma\left(\Lambda_{M^{\prime}}\right)-\Gamma\left(\Lambda_{M^{\prime \prime}}\right)\right|^{2} d \mu
$$

or

$$
\left\|U_{M^{\prime}} f-U_{M^{\prime \prime}} f\right\| \leqslant \frac{K}{2 \sigma^{2}}\left\|\Gamma\left(\Lambda_{M^{\prime}}-\Lambda_{M^{\prime \prime}}\right)\right\|
$$

From this, we infer by Theorem 1.4 and Theorem 2.5 that the net $\left(U_{M} f: M \in\right.$ $\mathscr{F}(L))$ is Cauchy; let us write $U_{\Lambda} f$ for its limit it $\mathbb{H}(L)$. Observe not only that $\left\|U_{\Lambda} f\right\|=\|f\|$ but also that if $M \in \mathscr{F}(L)$ then

$$
\left\|U_{\Lambda} f-U_{\Lambda_{M}} f\right\| \leqslant \frac{K}{2 \sigma^{2}}\left\|\Gamma\left(\Lambda-\Lambda_{M}\right)\right\| .
$$

It is a familiar fact that if $M \in \mathscr{F}(L)$ then $H(M)$ has its subset $H_{0}(M)$ of bounded elements as a dense subspace; consequently, the union

$$
H_{0}(L)=\bigcup\left\{H_{0}(M): M \in \mathscr{F}(L)\right\}
$$

is a dense subspace of $\mathbb{H I}(L)$. It follows that the linear isometry $U_{\Lambda}: H_{0}(L) \rightarrow$ $\mathbb{H}(L)$ constructed above extends uniquely to a linear isometry $U_{A}: \mathbb{H}(L) \rightarrow$ $\mathbb{H}(L)$. Observe that if $f \in \mathbb{H}(L)$ then the net $\left(U_{\Lambda_{M}} f: M \in \mathscr{F}(L)\right)$ converges to $U_{\Lambda} f$ : indeed, if $\varepsilon>0$ is given and if $f_{0} \in H_{0}(L)$ is chosen so that $\left\|f-f_{0}\right\| \leqslant \varepsilon$ then

$$
\begin{aligned}
& \left\|U_{\Lambda} f-U_{\Lambda_{M}} f\right\| \\
& \quad \leqslant\left\|U_{\Lambda} f-U_{\Lambda} f_{0}\right\|+\left\|U_{\Lambda} f_{0}-U_{\Lambda_{M}} f_{0}\right\|+\left\|U_{\Lambda_{M}} f_{0}-U_{\Lambda_{M}} f\right\| \\
& \quad \leqslant 2 \varepsilon+\left\|U_{\Lambda} f_{0}-U_{\Lambda_{M}} f_{0}\right\|
\end{aligned}
$$

and we need only recall that the net $\left(U_{\Lambda_{M}} f_{0}: M \in \mathscr{F}(L)\right)$ converges to $U_{A} f_{0}$. 
Theorem 2.7. The assignment

$$
U: \mathbb{P} L \rightarrow \operatorname{Aut} \mathbb{\mathbb { H }}(L): \Lambda \mapsto U_{\Lambda}
$$

is a group homomorphism relative to addition in $\mathbb{P} L$ and composition in $\operatorname{Aut} \mathbb{H}(L)$.

Proof. Let $\Lambda^{\prime}, \Lambda^{\prime \prime} \in \mathbb{P} L$ and let $f \in H(L)$. On the one hand, $U_{\Lambda^{\prime}{ }_{M}} U_{\Lambda^{\prime \prime}{ }_{M}} f \longrightarrow$ $U_{\Lambda^{\prime}} U_{\Lambda^{\prime \prime}} f$ because composition of isometries is continuous relative to the strong operator topology: explicitly, if $\varepsilon>0$ is given and if $M_{\varepsilon} \in \mathscr{F}(L)$ is chosen so that $M_{\varepsilon} \subset M \in \mathscr{F}(L)$ implies both $\left\|\left(U_{\Lambda^{\prime \prime} M}-U_{\Lambda^{\prime \prime}}\right) f\right\| \leqslant \varepsilon$ and $\left\|\left(U_{\Lambda^{\prime} M}-U_{\Lambda^{\prime}}\right) U_{\Lambda^{\prime \prime}} f\right\| \leqslant \varepsilon$ then it follows that

$$
\begin{gathered}
\left\|U_{\Lambda^{\prime}{ }_{M}} U_{\Lambda^{\prime \prime}{ }_{M}} f-U_{\Lambda^{\prime}} U_{\Lambda^{\prime \prime}} f\right\| \\
\leqslant\left\|U_{\Lambda^{\prime}{ }_{M}}\left(U_{\Lambda^{\prime \prime}{ }_{M}}-U_{\Lambda^{\prime \prime}}\right) f\right\|+\left\|\left(U_{\Lambda^{\prime}{ }_{M}}-U_{\Lambda^{\prime}}\right) U_{\Lambda^{\prime \prime}} f\right\| \leqslant 2 \varepsilon .
\end{gathered}
$$

On the other hand, $U_{\Lambda_{M}^{\prime}} U_{\Lambda_{M}^{\prime \prime}} f \rightarrow U_{\Lambda^{\prime}+\Lambda^{\prime \prime}} f:$ indeed,

$$
\begin{aligned}
U_{\Lambda^{\prime}{ }_{M}} U_{\Lambda_{M}} f & =\exp \left\{\frac{\Gamma\left(\Lambda_{M}^{\prime}\right)+\Gamma\left(\Lambda_{M}^{\prime \prime}\right)}{2 i \sigma^{2}}\right\} f \\
& =\exp \left\{\frac{\Gamma\left(\left(\Lambda^{\prime}+\Lambda^{\prime \prime}\right)_{M}\right)}{2 i \sigma^{2}}\right\} f \\
& =U_{\left(\Lambda^{\prime}+\Lambda^{\prime \prime}\right)_{M}} f .
\end{aligned}
$$

The equality $U_{\Lambda^{\prime}} U_{\Lambda^{\prime \prime}}=U_{\Lambda^{\prime}+\Lambda^{\prime \prime}}$ holds thus on $H(L)$ and so on $\mathbb{H}(L)$. It follows that if $\Lambda \in \mathbb{P} L$ then $U_{\Lambda}: \mathbb{H}(L) \rightarrow \mathbb{H}(L)$ is unitary with $U_{-\Lambda}$ as inverse, since $U_{0}=$ I.

The unitary representation $U$ of $\mathbb{P} L$ on $\mathbb{R}(L)$ is continuous in the following sense.

Theorem 2.8. Equipping $\mathbb{P} L$ with its Hilbert-Schmidt topology and Aut $\mathbb{H I}(L)$ with its strong operator topology, the map $U: \mathbb{P} L \rightarrow A u t \mathbb{Z}(L)$ is continuous on $\bigoplus_{j=0}^{d} \mathbb{P}^{j} L$ for each $d \in \mathbb{N}$.

Proof. As usual, we need only check continuity at zero. For this, let $\Lambda \in \mathbb{P} L$ and let $f_{0} \in H_{0}(L)$ with $K_{0}=\sup \left|f_{0}\right|$. In the inequality

$$
\left\|U_{\Lambda} f_{0}-U_{\Lambda_{M}} f_{0}\right\| \leqslant \frac{K_{0}}{2 \sigma^{2}}\left\|\Gamma\left(\Lambda-\Lambda_{M}\right)\right\|
$$

we let $M \in \mathscr{F}(L)$ be the zero subspace, yielding

$$
\left\|U_{\Lambda} f_{0}-f_{0}\right\| \leqslant \frac{K_{0}}{2 \sigma^{2}}\|\Gamma(\Lambda)\|
$$

Now, let $f \in \mathbb{H}(L)$ be arbitrary and let $\varepsilon>0$ : if $f_{0} \in H_{0}(L)$ satisfies $\left\|f-f_{0}\right\| \leqslant \varepsilon$ and 
if $\Lambda \in \mathbb{P} L$ satisfies $\|\Gamma(\Lambda)\| \leqslant \frac{2 \sigma^{2}}{K_{0}} \varepsilon$ then

$$
\left\|U_{\Lambda} f-f\right\| \leqslant\left\|U_{\Lambda} f-U_{\Lambda} f_{0}\right\|+\left\|U_{\Lambda} f_{0}-f_{0}\right\|+\left\|f_{0}-f\right\| \leqslant 3 \varepsilon
$$

the continuity of $\Gamma$ as expressed in the discussion following Theorem 2.5 concludes the proof.

Here, restriction to subspaces of bounded degree is occasioned by the fact that if $d \in \mathbb{N}$ then only $\sigma^{-d} \Gamma: \mathbb{P}^{d} L \rightarrow \mathbb{H}(L)$ is isometric. If for each $d \in \mathbb{N}$ the factor $\sigma^{d}$ is absorbed in the inner product on $P^{d} L$ by rescaling, then the corresponding map $\Gamma: \mathbb{P} L \rightarrow \mathbb{H}(L)$ is isometric and the resulting map $U: \mathbb{P} L \rightarrow$ $\operatorname{Aut} \mathbb{H}(L)$ is Hilbert-Schmidt-strong continuous under no restriction. In any case, it is certainly true for instance that if $\Lambda \in \mathbb{P} L$ is fixed then the one-parameter unitary group $\left(U_{t \Lambda}: t \in \mathbb{R}\right)$ on $\mathbb{H}(L)$ is strongly continuous.

We close this section by showing that on tame polynomials, the Wick transform commutes with translations.

Theorem 2.9. If $\Lambda \in P L$ and if $u \in L$ then

$$
T^{u} \Gamma(\Lambda)=\Gamma T^{u}(\Lambda) .
$$

Proof. Direct calculation. Choose $M \in \mathscr{F}(L)$ so that $\Lambda$ is based on $M$ and $M$ contains $u$. If $z \in L$ then

$$
\begin{aligned}
T^{u} \Gamma(\Lambda)(z) & =\Gamma(\Lambda)(u+z) \\
& =\int_{M} \Lambda(u+z+i w) d \mu(w) \\
& =\int_{M} T^{u} \Lambda(z+i w) d \mu(w) \\
& =\Gamma T^{u}(\Lambda)(z)
\end{aligned}
$$

Of course, it follows as a direct consequence that if $\Lambda \in P L$ and if $u \in L$ then

$$
\Gamma(\Lambda)^{u}=\Gamma\left(\Lambda^{u}\right)
$$

\section{Polynomial Weyl Representations}

Once again, $L$ is a real Hilbert space with $(\cdot \mid \cdot)$ as its inner product. We equip the direct sum $V=L \oplus L$ with the real symplectic form $\Omega$ defined by the rule that if $x_{1}, x_{2}, y_{1}, y_{2} \in L$ then

$$
\Omega\left(x_{1} \oplus y_{1}, x_{2} \oplus y_{2}\right)=\left(x_{1} \mid y_{2}\right)-\left(y_{1} \mid x_{2}\right)
$$

and with the compatible complex structure 


$$
J: V \rightarrow V: x \oplus y \mapsto(-y) \oplus x
$$

so that $V$ becomes a complex Hilbert space whose complex inner product $\langle\bullet \mid \cdot\rangle$ has $\Omega$ as its imaginary part: thus, if $v_{1}, v_{2} \in V$ then

$$
\left\langle v_{1} \mid v_{2}\right\rangle=\Omega\left(v_{1}, J v_{2}\right)+i \Omega\left(v_{1}, v_{2}\right) .
$$

Note that each complex Hilbert space may be regarded as arising in this way: if $V$ is a complex Hilbert space, then the points fixed by a conjugation constitute an appropriate real subspace $L$ from which it may be recovered.

By a (regular) Weyl representation of the real symplectic vector space $(V$, $\Omega$ ) we mean a unitary projective representation $W: V \rightarrow$ Aut $\mathbb{H}$ of the additive group underlying $V$ on a complex Hilbert space $\mathbb{H}$ having (Weyl) cocycle given by the rule that if $v_{1}, v_{2} \in V$ then

$$
W\left(v_{1}\right) W\left(v_{2}\right)=\exp \left\{\frac{\Omega\left(v_{1}, v_{2}\right)}{2 i \hbar}\right\} W\left(v_{1}+v_{2}\right)
$$

and having the (regularity) property that if $v \in V$ then the one-parameter unitary group $(W(t v): t \in \mathbb{R})$ is strongly continuous. Here, $\hbar=h / 2 \pi$ is a positive real number to be interpreted as the Planck constant. Regularity ensures a corresponding Heisenberg representation: to each $v \in V$ is associated the self-adjoint infinitesimal generator $\Phi(v)$ of $(W(t \sqrt{\hbar} v): t \in \mathbb{R})$ so that if $t \in$ $\mathbb{R}$ then $W(t \sqrt{\hbar} v)=\exp \{i t \Phi(v)\}$; these field operators $\{\Phi(v): v \in V\}$ satisfy the canonical commutation relations in Heisenberg form, so that if $v_{1}, v_{2} \in V$ then

$$
\left[\Phi\left(v_{1}\right), \Phi\left(v_{2}\right)\right] \subset i \Omega\left(v_{1}, v_{2}\right) I \text {. }
$$

The complex structure $J$ distinguishes a canonical irreducible Weyl representation of $(V, \Omega)$ : its Fock representation, which has several equivalent versions. We find it convenient to work with the version known as the renormalized Schrödinger (or real wave) representation, of which we offer a brief account. Henceforth, the variance of the Gaussian distribution component of $Q$-space will have the specific value $\sigma^{2}=\frac{1}{2} \hbar$.

The renormalized Schrödinger representation of $V$ is carried by $Q$-space over $L$ and will be denoted by $W_{0}: V \rightarrow \operatorname{Aut} \mathbb{H I}(L)$. Explicitly, if $x, y \in L$ then $W_{0}(x \oplus y)$ is defined as follows. If $f \in H(L)$ is a tame function on $L$ then $W_{0}(x \oplus$ y) $f \in H(L)$ is defined by the rule that if $z \in L$ then

$$
\left[W_{0}(x \oplus y) f\right](z)=\exp \left[\frac{(x \mid 2 z-y)}{2 i \hbar}+\frac{(y \mid 2 z-y)}{2 \hbar}\right] f(z-y) .
$$

Note that $W_{0}(x \oplus y) f$ is indeed tame: if $f$ is based on $M \in \mathscr{F}(L)$ then $W_{0}(x \oplus y) f$ is based on $M+\mathbb{R} x+\mathbb{R} y \in \mathscr{F}(L)$. The resulting map $W_{0}(x \oplus y): H(L) \rightarrow H(L)$ is isometric; the term in the exponent compensates for the fact that the Gaussian measures are only quasi-invariant under translation. A routine algebraic 
calculation shows that $W_{0}: V \rightarrow \operatorname{End} H(L)$ so defined has the Weyl cocycle, whence each $W_{0}(x \oplus y): H(L) \rightarrow H(L)$ is actually an isometric isomorphism which therefore extends to a unitary operator $W_{0}(x \oplus y): \mathbb{H I}(L) \rightarrow \mathbb{H I}(L)$. Finally, the resulting map $W_{0}: V \rightarrow \operatorname{Aut} \mathbb{H}(L)$ continues to have the Weyl cocycle and is readily verified to be regular.

Starting from the renormalized Schrödinger representation, we fashion a new Weyl representation of $V$ on $Q^{-}$-space as follows. First, let $\Lambda: L \rightarrow \mathbb{R}$ be a differentially Hilbert-Schmidt polynomial: a polynomial on $L$ such that if $u \in L$ then $\Lambda^{u} \in \mathbb{P} L$ is Hilbert-Schmidt; recall that $\Lambda^{u}: L \rightarrow \mathbb{R}$ is the polynomial with value $\Lambda^{u}(z)=\Lambda(u+z)-\Lambda(z)$ at $z \in L$. Let $U_{\Lambda^{u}} \in \operatorname{Aut} \mathbb{H}(L)$ be the unitary operator constructed for Theorem 2.7; in particular, if $\Lambda^{u}$ is tame then $U_{\Lambda^{u}}$ acts on $f \in H(L)$ according to

$$
U_{\Lambda}^{u f}=\exp \left[\frac{\Gamma\left(\Lambda^{u}\right)}{i \hbar}\right] f
$$

Now, to each $x, y \in L$ let us associate the unitary operator $W_{\Lambda}(x \oplus y) \in \operatorname{Aut} \mathbb{H I}(L)$ defined by the formula

$$
W_{\Lambda}(x \oplus y)=W_{0}(x \oplus y) U_{\Lambda^{y}}
$$

Theorem 3.1. $W_{A}: V \rightarrow A u t \mathbb{H I}(L)$ is a Weyl representation.

Proof. We must confirm that $W_{A}$ is regular and has the Weyl cocycle. Regularity is straightforward: on the one hand, the renormalized Schrödinger representation $W_{0}$ itself regular; on the other, representation if $u \in L$ then the map $\mathbb{R} \rightarrow \operatorname{Aut} \mathbb{H}(L): t \mapsto U_{A^{t u}}$ is strongly continuous on account of Theorem 2.8 and the fact that $\mathbb{R} \rightarrow \mathbb{P} L: t \mapsto \Lambda^{t u}$ is continuous. Before checking that $W_{\Lambda}$ has the correct cocycle, it is convenient to note that if $u \in M \in \mathscr{F}(L)$ then $\left(\Lambda^{u}\right)_{M}=\left(\Lambda_{M}\right)^{u}$ and that in discussing net convergence we may confine our attention to such $M$ as contain $u$. Thus, if $x, y \in L$ then the unitary $W_{\Lambda_{M}}(x \oplus y)$ converges strongly to $W_{\Lambda}(x \oplus y)$ as $M$ runs over the directed set $\{M \in \mathscr{F}(L): y \in M\}$. Similarly, if $x_{1}, x_{2}$, $y_{1}, y_{2} \in L$ then

$$
W_{\Lambda_{M}}\left(x_{1} \oplus y_{1}\right) W_{\Lambda_{M}}\left(x_{2} \oplus y_{2}\right) \rightarrow W_{\Lambda}\left(x_{1} \oplus y_{1}\right) W_{\Lambda}\left(x_{2} \oplus y_{2}\right)
$$

in the strong operator topology as $M$ runs over $\left\{M \in \mathscr{F}(L): y_{1}, y_{2} \in M\right\}$. Now, direct calculation from the definitions shows that if $y_{1}, y_{2} \in M \in \mathscr{F}(L)$ then on $H(L)$ there holds the equality

$$
W_{\Lambda_{M}}\left(x_{1} \oplus y_{1}\right) W_{\Lambda_{M}}\left(x_{2} \oplus y_{2}\right)=\exp \left[\frac{\Omega\left(x_{1} \oplus y_{1}, x_{2} \oplus y_{2}\right)}{2 i \hbar}+\frac{\Delta}{i \hbar}\right] W_{\Lambda_{M}}\left(\left(x_{1} \oplus y_{1}\right)+\left(x_{2} \oplus y_{2}\right)\right)
$$

where $\Delta: L \rightarrow \mathbb{R}$ is given by 


$$
\begin{aligned}
\Delta & =T^{-y_{1}} \Gamma\left(\Lambda_{M}^{y_{1}}\right)+T^{-y_{1}-y_{2}} \Gamma\left(\Lambda_{M}^{y_{2}}\right)-T^{-y_{1}-y_{2}} \Gamma\left(\Gamma_{M}^{y_{1}+y_{2}}\right) \\
& =\Gamma\left(T^{-y_{1}} \Lambda_{M}^{y_{1}}+T^{-y_{1}-y_{2}} \Lambda_{M}^{y_{2}}-T^{-y_{1}-y_{2}} \Lambda_{M}^{y_{1}+y_{2}}\right)=0
\end{aligned}
$$

thanks to Theorem 2.9; by continuity, the same equality holds on $\mathbb{B}(L)$. Passage to the limit as $M$ runs over $\left\{M \in \mathscr{F}(L): y_{1}, y_{2} \in M\right\}$ now yields the fact that $W_{\Lambda}$ has the Weyl cocycle.

We shall refer to $W_{\Lambda}$ as a polynomial Weyl representation: specifically, as the polynomial Weyl representation generated by $\Lambda$. Observe that our notation is consistent: if $\Lambda=0$ then $W_{\Lambda}$ is simply the renormalized Schrödinger representation.

It is natural to ask for the extent to which the Weyl representation $W_{\Lambda}$ varies as the polynomial $\Lambda$ itself is varied. An almost immediate consequence of our construction is that up to unitary equivalence, $W_{A}$ is unaffected by Hilbert-Schmidt perturbations of $\Lambda$.

Theorem 3.2. If $\Lambda: L \rightarrow \mathbb{R}$ is a differentially Hilbert-Schmidt polynomial and if $\Delta \in \mathbb{P} L$ then the Weyl representations $W_{\Lambda}$ and $W_{\Lambda+\Delta}$ are unitarily equivalent: indeed, if $x, y \in L$ then

$$
W_{\Lambda+\Delta}(x \oplus y)=U_{\Delta} W_{\Lambda}(x \oplus y) U_{\Delta}^{-1} .
$$

Proof. Of course, the polynomial $\Lambda+\Delta$ is differentially Hilbert-Schmidt and the unitary operator $U_{\Delta}$ is defined according to Theorem 2.7. If $y \in M \in \mathscr{F}(L)$ then direct calculation using Theorem 2.9 shows that

$$
U_{\Delta_{M}} W_{0}(x \oplus y) U_{\Lambda_{M}^{y}} U_{\Delta_{M}}^{-1}=W_{0}(x \oplus y) U_{(\Lambda+\Delta)_{M}^{y}}
$$

on $H(L)$ and therefore on $\mathbb{H I}(L)$. Thanks to the strong operator continuity on unitaries of multiplication and inversion, passage to the limit as $M \rightarrow L$ concludes the proof.

Otherwise said, let $\Lambda^{\prime}$ and $\Lambda^{\prime \prime}$ be differentially Hilbert-Schmidt polynomials on $L$ : if the difference $\Lambda^{\prime \prime}-\Lambda^{\prime}$ is Hilbert-Schmidt, then the representations $W_{\Lambda^{\prime}}$ and $W_{\Lambda^{\prime \prime}}$ are unitarily equivalent. We conjecture the truth of the converse: that if $W_{\Lambda^{\prime}}$ and $W_{\Lambda^{\prime \prime}}$ are unitarily equivalent then $\Lambda^{\prime \prime}-\Lambda^{\prime}$ is Hilbert-Schmidt. In particular, let $\Lambda: L \rightarrow \mathbb{R}$ be a differentially Hilbert-Schmidt polynomial. We conjecture that if $W_{A}$ is unitarily equivalent to the renormalized Schrödinger representation $W_{0}$ then $\Lambda \in \mathbb{P} L$.

Evidence for this conjecture is provided by the following argument. First, if $v \in V$ then let $\stackrel{\circ}{W}_{\Lambda}(v)$ denote the skew-adjoint infinitesimal generator of the strongly continuous one-parameter unitary group $\left(W_{\Lambda}(t v): t \in \mathbb{R}\right)$ so that $W_{\Lambda}(v)=\exp \stackrel{D}{W}_{\Lambda}(v)$. By definition, a Fock vacuum for $W_{\Lambda}$ is a nonzero vector in the domain of and annihilated by the operator $\stackrel{\circ}{W}_{\Lambda}(v)+i W_{\Lambda}(J v)$ whenever $v \in V$. 
In particular, the Fock vacua for the renormalized Schrödinger representation $W_{0}$ itself are precisely the nonzero constant functions $L \rightarrow \mathbb{C}$. Now, let $U \in$ AutiHI $(L)$ intertwine $W_{0}$ and $W_{A}$ so that

$$
v \in V \Rightarrow W_{\Lambda}(v)=U W_{0}(v) U^{-1} .
$$

Application of $U$ to a Fock vacuum for $W_{0}$ yields a Fock vacuum $f \in \mathbb{H}(L)$ for $W_{\Lambda}$. Informal solution of the differential equations

$$
v \in V \Rightarrow\left[\stackrel{\circ}{W}_{\Lambda}(v)+i \stackrel{\circ}{W}_{\Lambda}(J v)\right] f=0
$$

shows that $f$ is a scalar multiple of the function $\exp [\Gamma(\Lambda) / i \hbar]$ and places $\Lambda$ in $\mathbb{P} L$. Unfortunately, the technical details involved in promoting this to a formal argument are less than straightforward.

It is instructive to examine some low degree polynomial Weyl representations. For simplicity, we shall let $\Lambda: L \rightarrow \mathbb{R}$ be a differentially Hilbert-Schmidt $(d+1)$-homogeneous polynomial and consider the cases $d \in\{0$, $1,2\}$ explicitly.

Example $d=0$. In this case, $\Lambda: L \rightarrow \mathbb{R}$ is an arbitrary linear functional and the differentially Hilbert-Schmidt condition is redundant. If $u \in M \in \mathscr{F}(L)$ and if $z \in L$ then $\Lambda_{M}^{u}(z)=\Lambda_{M}(u)=\Lambda(u)$ so that $\Gamma\left(\Lambda_{M}^{u}\right)(z)=\Lambda(u)$ and therefore

$$
f \in \mathbb{H}(L) \Rightarrow U_{\Lambda^{u}} f=\exp \left[\frac{\Lambda(u)}{i \hbar}\right] f .
$$

Accordingly, if $x, y \in L$ then

$$
W_{\Lambda}(x \oplus y)=W_{0}(x \oplus y) \exp \left[\frac{\Lambda(y)}{i \hbar}\right]
$$

so that the Weyl representation $W_{\Lambda}$ belongs to the familiar class of coherent Weyl representations. It is known that $W_{A}$ is unitarily equivalent to $W_{0}$ if and only if $\Lambda$ is bounded if and only if $\Lambda \in \mathbb{P}^{1} L$. See [3].

Example $d=1$. In this case, Theorem 1.9 tells us that $\Lambda$ is bounded and so corresponds to a symmetric bounded linear operator $Z: L \rightarrow L$ according to the rule

$$
x, y \in L \Rightarrow \underline{\Lambda}(x, y)=\frac{1}{2}(x \mid Z y)
$$

When $M \in \mathscr{F}(L)$ we shall write $Z_{M}$ for the compression $P_{M} Z P_{M}$ so that

$$
x, y \in L \Rightarrow \underline{\Lambda}_{M}(x, y)=\frac{1}{2}\left(x \mid Z_{M} y\right) .
$$

If $u \in M \in \mathscr{F}(L)$ and if $z \in L$ then 


$$
\Gamma\left(\Lambda_{M}^{u}\right)(z)=\Lambda_{M}^{u}(z)=\left(z \mid Z_{M} u\right)+\frac{1}{2}(u \mid Z u)
$$

so that if also $f \in H(L)$ then

$$
\left[U_{\Lambda_{M}^{u} f}\right](z)=\exp \left[\frac{\left(z \mid Z_{M u}\right)}{i \hbar}+\frac{(u \mid Z u)}{2 i \hbar}\right] f(z) .
$$

Now, let $x, y \in L$ and stipulate that $M \in \mathscr{F}(L)$ contain both $y$ and $Z y$ whence $Z_{M} y$ $=Z y$. If $f \in H(L)$ and $z \in L$ then

$$
\left[W_{0}(x \oplus y) U_{\Lambda_{M}^{y}} f\right](z)=\exp \left[\frac{(x \mid 2 z-y)}{2 i \hbar}+\frac{(y \mid 2 z-y)}{2 \hbar}+\frac{(z \mid Z y)}{i \hbar}-\frac{(z \mid Z y)}{2 i \hbar}\right] f(z-y)
$$

from which it is plain upon passage to the limit as $M \rightarrow L$ that

$$
W_{\Lambda}(x \oplus y)=W_{0}((x+Z y) \oplus y) .
$$

It is readily confirmed that the map

$$
g: V \rightarrow V: x \oplus y \mapsto(x+Z y) \oplus y
$$

is an automorphism of $(V, \Omega)$ as a real symplectic vector space. Accordingly, $W_{A}=W_{0} \circ \mathrm{g}$ is again one of a familiar class of Weyl representations: it arises from the Fock representation $W_{0}$ via precomposition by a symplectic automorphism. In fact, $W_{A}=W_{0} \circ g$ is just a version of the Fock representation of $(V, \Omega)$ distinguished by the complex structure $\mathrm{gJg}^{-1}$. A celebrated result of Shale implies that $W_{0} \circ g$ is unitarily equivalent to $W_{0}$ if and only if $Z$ is Hilbert-Schmidt, so that $W_{\Lambda}$ is unitarily equivalent to $W_{0}$ if and only if $\Lambda \in \mathbb{P}^{2} L$. See [11].

In the next example, Wick-ordering makes its presence felt for the first time; by way of preparation, we insert the following basic formula.

Theorem 3.3. Let $\Lambda \in P^{2} L$ and let $Z: L \rightarrow L$ be the symmetric linear operator of finite rank satisfying

$$
x, y \in L \Rightarrow \underline{\Lambda}(x, y)=(x \mid Z y) .
$$

If $z \in L$ then

$$
\Gamma(\Lambda)(z)=(z \mid Z z)-\frac{1}{2} \hbar \operatorname{Tr} Z
$$

Proof. Direct calculation from the difinitions. The tame polynomial $\Lambda$ is based on the range $M \in \mathscr{F}(L)$ of $Z$ and

$$
\begin{aligned}
\Gamma(\Lambda)(z)=\int_{M} \Lambda(z+i w) d \mu(w) & =\int_{M}\{(z \mid Z z)+2 i(z \mid Z w)-(w \mid Z w)\} d \mu(w) \\
& =(z \mid Z z)-\int_{M}(w \mid Z w) d \mu(w)
\end{aligned}
$$


This last integral may be evaluated by diagonalization, for example: if $Z$ on $M$ has eigenvalues $\lambda_{1}, \cdots, \lambda_{m}$ repeated by multiplicity, then

$$
\begin{aligned}
\int_{M}(w \mid Z w) d \mu(w) & =\sum_{j=1}^{m} \lambda_{j} \int_{\mathbb{R}} t^{2} d \mu(t) \\
& =\frac{1}{2} \hbar \operatorname{Tr} Z
\end{aligned}
$$

since $\mu$ is the law of a random variable with $N\left(0, \frac{1}{2} \hbar\right)$ distribution.

Example $d=2$. In this case, Theorem 1.9 again informs us that $\Lambda$ is bounded. Thus, the rule

$$
x, y, z \in L \Rightarrow \underline{\Lambda}(x, y, z)=\frac{1}{3}\left(x \mid Z^{z} y\right)
$$

defines a bounded linear map $Z$ associating to each $z \in L$ a symmetric Hilbert-Schmidt operator $Z^{z}: L \rightarrow L$ and having the property that $Z^{x} y=Z^{y} x$ whenever $x, y \in L$. Note that if $M \in \mathscr{F}(L)$ and $x, y, z \in L$ then

$$
\Lambda(x, y, z)=\frac{1}{3}\left(x \mid Z_{M}^{P_{M} z} y\right) .
$$

Consequently, if $u \in L$ and if $M \in \mathscr{F}(L)$ is chosen to contain both $u$ and $Z^{u} u$ then for each $z \in L$

$$
\Lambda_{M}^{u}(z)=\frac{1}{3}\left(u \mid Z^{u} u\right)+\left(z \mid Z^{u} u\right)+\left(z \mid Z_{M z}^{u}\right)
$$

and therefore

$$
\Gamma\left(\Lambda_{M}^{u}\right)(z)=\Lambda_{M}^{u}(z)-\frac{1}{2} \hbar \operatorname{Tr} Z_{M}^{u}
$$

according to Theorem 3.3. It follows from this that if also $f \in H(L)$ then

$$
\left[U_{\Lambda_{M}^{u} f}\right](z)=\exp \left[\frac{\left(u \mid Z^{u} u\right)}{3 i \hbar}+\frac{\left(z \mid Z^{u} u\right)}{i \hbar}+\frac{\left(z \mid Z_{M}^{u} z\right)}{i \hbar}+\frac{1}{2} i \operatorname{Tr} Z_{M}^{u}\right] f(z)
$$

For obvious reasons, we shall not insist on exhibiting a similarly explicit formula for the resulting Weyl representation $W_{\Lambda}$. As a matter of fact, this $W_{\Lambda}$ belongs to an unfamiliar class of Weyl representations. In fact $W_{A}$ is precisely a quadratic Weyl representation as presented in [8]. It is known that the quadratic Weyl representation $W_{A}$ is unitarily equivalent to the renormalized Schrödinger representation $W_{0}$ if and only if $\Lambda \in \mathbb{P}^{3} L$ is Hilbert-Schmidt. See $[7]$. 
A remark is in order here. In [8] we constructed a quadratic Weyl representation in terms of the metaplectic representation engendered by the renormalized Schrödinger representation. There, each quadratic Weyl operator was expressed as the product of three unitaries: a renormalized Schrödinger Weyl operator precomposed with a symplectic automorphism; the square of an element of the metaplectic group implementing the same symplectic automorphism; and a scalar factor (the exponential of a imaginary cubic) which had to be inserted by hand in order to satisfy the Weyl cocycle. An important aspect of the approach taken in the present paper is that the somewhat mysterious unitary scalar factor is automatically incorporated in the construction.

\section{Discussion}

Our account of polynomial Weyl representations raises a number of questions, some of which we discuss briefly in this final section.

First of all, it is reasonable to ask for a simplification of the technical details involved in establishing our conjecture that if the representations $W_{\Lambda^{\prime}}$ and $W_{\Lambda^{\prime \prime}}$ are unitarily equivalent then the difference between $\Lambda^{\prime}$ and $\Lambda^{\prime \prime}$ is actually Hilbert-Schmidt. A possible approach is to base polynomial Weyl representations not on the real wave representation but rather on the complex wave representation due to Segal. This alternative version of the Fock representation acts on a space of antiholomorphic functions square-integrable relative to a Gaussian distribution and has the technical advantage that each bounded linear operator on the carrier space is an integral operator, integration being in the functional sense. This circumstance suggests the possiblity of making intertwining operators between the complex wave representation and derived polynomial Weyl representations very explicit in terms of integral kernels. For the complex wave representation and its relation to the real wave representation via the (renormalized) Bargmann transform, we refer to [2].

It is natural to ask for an abstract characterization of polynomial Weyl representations, perhaps along the lines of known characterizations for Weyl representations in other classes. For example, the Fock representation associated to a complex structure may be characterized by the fact that its carrier space contains the vacuum as a cyclic vector in the kernel of each corresponding annihilator; it may also be characterized in terms of the number operator $N$ or the strongly continuous one-parameter unitary group $\left(e^{i t N}: t \in \mathbb{R}\right)$. The coherent representations and quasifree representations also have familiar characterizations: the former in terms of the vacuum as a common eigenvector for annihilators; the latter in terms of the vanishing of higher order truncated vacuum expectation values. For a variety of reasons, it would be helpful to have a characterization (in similar terms or otherwise) of polynomial Weyl 
representations by which to recognize them abstractly.

As noted earlier, if $g$ is a symplectic automorphism of $(V, \Omega)$ then $W_{0} \circ g$ is a version of the Fock representation corresponding to the complex structure $g J g^{-1}$. A celebrated theorem of Shale [11] implies that $W_{0} \circ g$ is unitarily equivalent to $W_{0}$ if and only if the commutator $g J-J g$ is a Hilbert-Schmidt operator. This leads directly to the construction of the metaplectic group and its metaplectic representation, of importance in numerous contexts. A natural project is to consider the effect of symplectic automorphisms on polynomial Weyl representations and in particular to determine precise necessary and sufficient conditions on the symplectic automorphism $g$ and the differentially Hilbert-Schmidt polynomial $\Lambda$ in order that the Weyl representations $W_{\Lambda} \circ g$ and $W_{\Lambda}$ be unitarily equivalent. Of course, an abstract characterization of polynomial Weyl representations is likely to be beneficial here.

The Weyl representations constructed in the present paper were generated by differentially Hilbert-Schmidt polynomials. We could consider extending the construction, replacing real polynomials by real analytic functions. For this purpose, it is probably convenient to absorb the variance $\sigma^{2}=\frac{1}{2} \hbar$ by appropriately rescaling the inner products between tame homogeneous polynomials, so that the Wick transform $\Gamma: P L \rightarrow \mathbb{H}(L)$ becomes an isometry and extends to an isometry on the Hilbert space completion $\overline{P L}$ each of whose elements may be regarded as a Hilbert-Schmidt analytic function on $L$. We may then consider the process of generating Weyl representations from $W_{0}$ by means of differentially Hilbert-Schmidt analytic functions: needless to say, we can expect that those generated by Hilbert-Schmidt analytic functions will be unitarily equivalent to $W_{0}$; the others should again be new.

Another direction in which to extend the present paper is to vary the basic representation. The Fock representation $W_{0}$ was chosen primarily on account of its technical convenience, it being relatively straightforward to handle rather explicitly. We may consider replacing the Fock representation by another Weyl representation and performing the same type of operation to generate further Weyl representations. For convenience, a quasifree representation might be chosen as basic: quasifree representations admit a standard description in terms of Fock representations by means of a doubling procedure; see [1] and [4]. When comparing Weyl representations in such a case, it would be sensible to replace the notion of unitary equivalence by the more general notion of quasiequivalence.

\section{References}

[1] Araki, H. and Shiraishi, M., On quasifree states of the canonical commutation relations (I), Publ. RIMS, Kyoto Univ., 7 (1971/72), 105-120. 
[2] Baez, J. C., Segal, I. E. and Zhou, Z., Introduction to Algebraic and Constructive Quantum Field Theory, Princeton University Press, 1992.

[3] Berezin, F. A., The Method of Second Quantization, Academic Press, 1966.

[4] van Daele, A., Quasi-equivalence of quasi-free states on the Weyl algebra, Comm. Math. Phys., 21 (1971), 171-191.

[5] Glimm, J. and Jaffe, A., Boson quantım field models, Mathematics of Contemporary Physics, Academic Press, (1972), 77-143.

[6] Kadison, R. V. and Ringrose, J. R., Fundamentals of the Theory of Operator Algebras, I, Academic Press, 1983.

[7] Proksch, M., Reents, G. and Summers, S. J., Quadratic representations of the canonical commutation relations, Publ. RIMS, Kyoto Univ., 31 (1995), 755-804.

[8] Robinson, P. L., Quadratic Weyl representations, University of Florida, preprint, 1996.

[9] Segal, I. E., Tensor algebras over Hilbert spaces I, Trans. Amer. Math. Soc., 81 (1956), 106-134.

[10] _ Mathematical Problems of Relativistic Physics, Amer. Math. Soc., 1963.

[11] Shale, D., Linear symmetries of free boson fields, Trans. Amer. Math. Soc., 103 (1962), 149-167.

[12] Simon, B., The $P(\phi)_{2}$ Euclidean (Quantum) Field Theory, Princeton University Press, 1974. 